\title{
Diagnostic des accès fébriles chez des enfants de la ville de Cabinda (Angola)
}

\author{
Diagnosis of Children's Fever Attacks in Health Facilities in the City of Cabinda (Angola)
}

\author{
M. Muila · P. Gazin \\ Reçu le 25 mars 2018; accepté le 25 mai 2018 \\ (C) Société de pathologie exotique et Lavoisier SAS 2018
}

Résumé Selon les recommandations de l'OMS, les traitements antipaludiques ne doivent être prescrits qu'en cas de confirmation biologique des accès palustres. Afin de mieux connaître la démarche diagnostique des accès fébriles chez les enfants ainsi que la prescription de médicaments antipaludiques, une enquête a été réalisée dans deux centres de santé de la ville de Cabinda (Angola) en 2014. Les diagnostics établis par les soignants et leurs prescriptions ont été analysés en fonction des résultats des tests rapides (TDR) et des examens sanguins microscopiques réalisés chez 787 enfants consultant ces centres pour fièvre. Le diagnostic de paludisme, seul ou associé à d'autres maladies, est établi chez la moitié des enfants bien qu'un TDR ne soit positif que chez $16 \%$ d'entre eux. Les soignants n'ont pas confiance dans le résultat des tests, en particulier en cas de négativité d'un TDR. Accès fébriles et accès palustres paraissent être souvent confondus en une même entité. Les autres causes de fièvre sont peu recherchées et les médicaments antipaludiques sont sur-prescrits. La formation des soignants et leur supervision par des médecins expérimentés sont nécessaires pour un bon usage des médicaments antipaludiques.

Mots clés Fièvre · Enfants · Paludisme · Diagnostic · TDR · Traitement $\cdot$ Cabinda $\cdot$ Angola $\cdot$ Afrique australe

\begin{abstract}
According to WHO recommendations, antimalarial drugs should be used only after a biological confirmation of a malaria attack. In order to better understand the diagnosis decision-making and the use of the malaria tests (immunochromatographic test RDT and direct microscope observation), a survey was carried out in two health facilities in the
\end{abstract}

M. Muila

Escola de formação de technicos de Saúde, Cabinda, Angola

P. Gazin $(\bowtie)$

Faculté de médecine et IRD, UMR 912, Marseille, France

e-mail : pierre.gazin@ird.fr city of Cabinda, Angola in 2014. Diagnosis and drug prescriptions by the health staff were observed in 787 febrile children. It clearly appeared that there was a lack of trust of the health professionals in the test results, especially in the RDT. They tended to take into account test results irregularly, giving a diagnosis of malaria for half the febrile children despite only $16 \%$ of positive RDT. Acute febrile illness and malaria attacks seemed thus frequently confused. Alternative causes for fever were rarely sought out. Antimalarial drugs were hence overprescribed. Better training for staff and of their supervision by experimented practitioners are one of the means envisaged for triggering real change in the providers' practice.

Keywords Fever - Children · Malaria · Diagnosis - RDT · Treatment $\cdot$ Cabinda $\cdot$ Angola $\cdot$ Southern Africa

\section{Introduction}

En Afrique au sud du Sahara, les accès de fièvres sont le motif le plus fréquent de consultation des enfants dans un centre de santé et le paludisme est une des principales causes de fièvre. Accès fébriles et accès palustres sont souvent confondus par les soignants ainsi que par les bénéficiaires de soins, entraînant une surestimation de la part du paludisme parmi les maladies infectieuses et un usage inapproprié des traitements antipaludiques.

En accord avec les recommandations de l'OMS, le ministère de la Santé en Angola préconise une confirmation du diagnostic de paludisme par un test rapide (TDR) ou par un examen microscopique du sang et le traitement des accès par Plasmodium falciparum par un antipaludique associant de l'artémisinine à une autre molécule efficace $[3,9]$. En pratique, comment le diagnostic de paludisme est-il effectué ? La situation dans deux centres de santé de la ville de Cabinda a été observée dans ce but. 


\section{Matériels et méthodes}

L'étude a été réalisée de juillet à octobre 2014 dans deux centres de santé de Cabinda : le Centro de Saude maternelinfantil et le Centro de Saude de Lombo Lombo. Ces centres de santé de base fournissent gratuitement des soins préventifs et curatifs pour les enfants et les femmes enceintes ou allaitantes. Les modalités de l'étude ont été établies en concertation avec les équipes de soignants. Elles ont été validées par la Direction provinciale de la santé de Cabinda.

L'enquêteur (MM) était présent une fois par semaine. Après information des adultes accompagnant les enfants sur l'objet de l'enquête et ses modalités, puis obtention de leur accord oral, les enfants fébriles (température axillaire $\geq$ $38,5^{\circ} \mathrm{C}$ ) ou avec une histoire récente de fièvre (moins de 24 heures) étaient inclus. Les refus de participer ont été rares et n'ont pas été comptabilisés. Pour chaque inclus, les soignants, le plus souvent des infirmiers, pratiquaient un test immuno-chromatographique rapide (One Step Malaria Anti-P.f (HRP-II)/P.v (pLDH) Cassette Test, InTec Products, Inc), une goutte épaisse selon leurs modalités habituelles ainsi qu'un frottis mince et une goutte épaisse supplémentaires pour un contrôle ultérieur. Les résultats des TDR étaient exprimés en présence ou absence de Plasmodium sans précision sur les espèces. Les gouttes épaisses étaient observées immédiatement et leurs résultats exprimés en présence ou absence de Plasmodium également sans précision sur les espèces. La décision de diagnostic et le choix de traitements étaient effectués par les soignants sans participation de l'enquêteur. Les médicaments antipaludiques, antipyrétiques et antibiotiques étaient disponibles dans les centres et délivrés gratuitement sans délai. Une lecture de contrôle d'un quart des frottis et gouttes épaisses supplémentaires a été effectuée ultérieurement à Marseille par un microscopiste expérimenté ne disposant pas des résultats produits à Cabinda.

\section{Résultats}

Au cours de l'enquête, 787 enfants consultant pour accès fébrile ont été inclus ( 377 filles et 410 garçons âgés de quelques mois au quinzième anniversaire). L'âge médian était de 2 ans et trois quarts des enfants étaient âgés de moins de 5 ans. Seize pour cent des TDR et $30 \%$ des gouttes épaisses observées dans les centres de santé étaient positifs (tableau 1). En cas de TDR négatif, $23 \%$ des gouttes épaisses étaient notées positives tandis que $31 \%$ des TDR positifs étaient associés à une goutte épaisse négative (faible degré de concordance, kappa de Cohen $=0,329[0,259-0,400]$ ).

Les principaux diagnostics, notés chez 723 enfants, ont été grippe, paludisme associé ou non à une autre maladie, « syndrome paludo-grippal » et infections respiratoires. Le
Tableau 1 Résultats des tests de diagnostic rapide (TDR) et des gouttes épaisses (GE) chez des enfants consultant pour accès fébrile dans deux centres de santé de Cabinda (Angola) en 2014 / RDT and thick smears (GE) results for children consulting for a fever episode in two health facilities in Cabinda (Angola) in 2014

\begin{tabular}{|llll|}
\hline & $\begin{array}{l}\text { GE négatives } \\
\text { selon les centres } \\
\text { de santé }\end{array}$ & $\begin{array}{l}\text { GE positives } \\
\text { selon les centres } \\
\text { de santé }\end{array}$ & Total \\
\hline TDR negatif & 509 & 154 & 663 \\
TDR positif & 39 & 85 & 124 \\
Total & 548 & 239 & 787 \\
\hline
\end{tabular}

« syndrome paludo-grippal» correspond à une absence de décision diagnostique entre une infection virale et un accès palustre. Ces diagnostics n'étaient pas liés aux résultats des tests parasitologiques (tableau 2). Le diagnostic de paludisme, associé ou non à une autre maladie, a été établi chez $33 \%$ des enfants avec une goutte épaisse négative et chez $42 \%$ de ceux avec un TDR négatif. Dix pour cent des enfants avec une goutte épaisse positive et $7 \%$ de ceux avec un TDR positif n'ont pas eu un diagnostic de paludisme.

Parmi les 272 diagnostics de paludisme dont le traitement est connu, $32 \%$ n'ont pas reçu de traitement antipaludique. Parmi les 153 diagnostics autres que paludisme, $3 \%$ ont reçu un traitement antipaludique. Le choix des traitements était-il lié aux résultats des examens parasitologiques ? En cas de TDR négatif, $54 \%$ des enfants recevaient l'association artemether-lumefantrine, $9 \%$ l'association artesunatesulfadoxine-pyriméthamine, $19 \%$ de la quinine et $16 \%$ de l'artemether. En cas de goutte épaisse négative, ces traitements étaient respectivement prescrits à $77 \%, 0 \%, 10 \%$ et $10 \%$ des enfants (tableau 3). L'association la plus utilisée, artemether-lumefantrine, a été prescrite à $51 \%$ des enfants avec un TDR positif, à $48 \%$ de ceux avec une goutte épaisse positive (tableau 3 ).

Les observations microscopiques de frottis et gouttes épaisses pour un contrôle ont confirmé $99 \%$ des résultats de TDR négatifs tandis que $60 \%$ des TDR positifs avaient un résultat microscopique négatif (tableau 4). P. falciparum a été la seule espèce observée lors de ces contrôles.

\section{Discussion}

Dans les deux centres de santé étudiés, la recherche de Plasmodium en cas de fièvre par TDR ou par examen d'une goutte épaisse est systématique. Le choix entre l'une ou l'autre technique dépend de la préférence de l'équipe soignante et de la disponibilité en matériel. Ce n'est que pour notre étude que les deux types de tests ont été systématiquement 
Tableau 2 Principaux diagnostics et résultats parasitologiques chez 723 enfants dans deux centres de santé de Cabinda (Angola) en 2014 / Main diagnoses made for 723 children in two health facilities in Cabinda (Angola) in 2014 and results of the parasitological tests

\begin{tabular}{|c|c|c|c|c|c|}
\hline & Grippe & Paludisme & $\begin{array}{l}\text { Syndrome paludo- } \\
\text { grippal }\end{array}$ & $\begin{array}{l}\text { Infection respiratoire } \\
\text { aiguë }\end{array}$ & Autres diagnostics \\
\hline \multirow{2}{*}{$\begin{array}{l}\text { Goutte épaisse } \\
\text { négative }\end{array}$} & 291 & 75 & 86 & 10 & 31 \\
\hline & $(59 \%)$ & $(15 \%)$ & $(17 \%)$ & $(2 \%)$ & $(6 \%)$ \\
\hline \multirow{2}{*}{$\begin{array}{l}\text { Goutte épaisse } \\
\text { positive }\end{array}$} & 20 & 145 & 61 & 1 & 3 \\
\hline & $(9 \%)$ & $(63 \%)$ & $(27 \%)$ & $(0,4 \%)$ & $(1 \%)$ \\
\hline \multirow[t]{2}{*}{ TDR négatif } & 304 & 124 & 130 & 11 & 33 \\
\hline & $(50 \%)$ & $(21 \%)$ & $(22 \%)$ & $(2 \%)$ & $(5 \%)$ \\
\hline \multirow[t]{2}{*}{ TDR positif } & 7 & 96 & 17 & 0 & 1 \\
\hline & $(6 \%)$ & $(79 \%)$ & $(14 \%)$ & $(0 \%)$ & $(1 \%)$ \\
\hline
\end{tabular}

Tableau 3 Traitements antipaludiques prescrits à 191 enfants dans deux centres de santé de Cabinda (Angola) en 2014 et résultats des examens parasitologiques. *Manalaria ${ }^{\circledR}$ est une polyphytothérapie dont l'activité antipaludique n'est pas connue / Antimalarial treatments given to 191 children in two health facilities in Cabinda (Angola) in 2014 and results of RDT and thick smears. * Manalaria ${ }^{\circledR}$ is a polyphytotherapy whose antimalarial action is not known

\begin{tabular}{|llllll|}
\hline & $\begin{array}{l}\text { Artemether }+ \\
\text { lumefantrine }\end{array}$ & Artesunate + SP & Quinine IV & Artemether IM & Manalaria®* \\
\hline TDR négatif & 61 & 10 & 21 & 18 & 3 \\
TDR positif & 40 & 1 & 28 & 7 & 2 \\
Goutte épaisse négative & 23 & 0 & 3 & 3 & 1 \\
Goutte épaisse positive & 78 & 11 & 46 & 22 & 4 \\
\hline
\end{tabular}

Tableau 4 Comparaison entre résultats des TDR et les examens microscopiques de contrôle chez 198 enfants consultant des centres de santé à Cabinda (Angola) en 2014. Densité parasitaire estimée par rapport aux leucocytes / Comparison between RDT and later microscopic observations of 198 children cared for in two health facilities in Cabinda (Angola) in 2014. Parasitological density related to leucocytes

\begin{tabular}{|lllll|}
\hline \multicolumn{4}{c}{ Frottis minces et gouttes épaisses observés en contrôle } & \\
\cline { 2 - 5 } & Négatif & Positifs $<2000 \mathrm{p} / \mu \mathrm{L}$ & Positifs $\geq 2000 \mathrm{p} / \mu \mathrm{L}$ & 0 \\
Total \\
\hline TDR négatif & 149 & 1 & 13 & 150 \\
TDR positif & 29 & 6 & 13 & 48 \\
Total & 178 & 7 & & 198 \\
\hline
\end{tabular}

effectués. Cependant, dans la pratique quotidienne, un test TDR négatif entraîne souvent la réalisation d'un examen microscopique complémentaire, les soignants n'ayant pas de confiance dans le premier résultat.

Au cours de l'étude, seulement $16 \%$ des 787 TDR étaient positifs mais le diagnostic de paludisme, seul ou associé à une autre maladie, a été porté chez $51 \%$ des enfants consultant les centres de santé. Parmi les enfants ayant un TDR ou une goutte épaisse négatifs, respectivement $42 \%$ et $33 \%$ ont été considérés comme souffrant de paludisme. À l'inverse,
$7 \%$ des TDR et $10 \%$ des gouttes épaisses positifs n'ont pas entraîné un diagnostic de paludisme. Pour un quart des TDR négatifs, les examens microscopiques effectués dans les centres de santé étaient considérés positifs. L'examen microscopique ultérieur de frottis et gouttes épaisses, avec un seul cas discordant, a infirmé ce résultat. Ce contrôle a par contre considéré que 29 TDR positifs parmi 48 étaient associés à un examen microscopique négatif. Cette divergence peut en partie être expliquée par la persistance de matériel parasitaire après la prise d'un traitement antipaludique efficace et 
également par un seuil de détection microscopique élevé pour des frottis et gouttes épaisses de réalisation inégale.

Les soignants effectuaient en principe un examen clinique des enfants, au moins sommaire. Cependant, cet examen était plutôt réservé aux enfants présentant un aspect inquiétant. Le choix des diagnostics et des traitements est resté durant l'étude sous la responsabilité des soignants. Cinquante neuf pour cent des 186 traitements antipaludiques réels ont été prescrits à des enfants avec un TDR négatif. La négativité d'un TDR n'a à l'évidence pas de signification pour de nombreux soignants. Ils semblent prendre leur décision diagnostique et thérapeutique principalement en fonction de leur impression clinique, de leur expérience et de leur conception de la maladie palustre. Accès fébrile, syndrome grippal et accès palustre apparaissent être confondus dans une seule entité symptomatique mal précisée entraînant une réponse uniforme.

Ce manque de confiance dans le résultat des examens parasitologiques, particulièrement en cas de négativité, peut être partiellement expliqué par le manque de formation sur l'utilisation des TDR et sur la signification de leurs résultats. Le choix de presque tout traiter comme du paludisme peut aussi être lié à l'absence d'autres outils de diagnostic. Il n'y a pas de test rapide disponible pour les autres principales étiologies de fièvre. Les soignants sont dans l'inconfortable situation d'être face à un grand nombre de consultants et de disposer de peu de techniques de diagnostic en dehors de l'interrogatoire et de l'examen clinique. La crainte de laisser évoluer un « léger paludisme » qui n'aurait pas été diagnostiqué par les tests vers une forme grave peut être comprise. À cela peut s'ajouter la persistance de la notion populaire de fièvre palustre imprégnant le corps sans hématozoaire détectable [2]. Enfin, les malades et leurs accompagnants sont demandeurs de traitements antipaludiques, des traitements qui, de plus, sont délivrés gratuitement et dont l'efficacité est connue. Tous ces éléments contribuent à un emploi peu rationnel des antipaludiques.

Des observations similaires ont été rapportées en Uganda, au Kenya, au Cameroun : des diagnostics erronés de paludisme et un emploi inapproprié des antipaludiques sont fréquents [1,4-6,8]. Une étude réalisée en 2010-2011 dans le centre de l'Angola a montré que le diagnostic de paludisme y était six fois plus fréquent que la positivité des gouttes épaisses [7].

La réalisation systématique d'un TDR ou d'un examen microscopique du sang en cas d'accès fébrile est-elle utile si leurs résultats ne sont pas pris en compte pour le diagnostic et pour le traitement? Dans des conditions de bonne pratique médicale, ces examens sont justifiés car ils permettent un emploi rationnel des antipaludiques et facilitent le diagnostic des autres pathologies [6]. La situation est différente là où leurs résultats ont peu d'effet sur le choix du diagnostic ou du traitement et en conséquence, ne réduisent pas l'excessive prescription d'antipaludiques. Cependant, l'abandon des tests, en particulier des TDR, pourrait aboutir à une situation encore pire que la situation actuelle.

Est-il possible d'améliorer les pratiques de soins ? La signification des tests, en particulier de la négativité d'un TDR, doit être davantage expliquée aux soignants et l'examen clinique valorisé. Une meilleure pratique de soins passe également par l'amélioration des conditions de travail, avec moins de consultants par professionnel incitant à plus de réflexion pour chaque cas. Formation professionnelle, supervision par des encadrants expérimentés et meilleures conditions de travail des soignants dans les centres de santé de base sont essentiels pour un usage rationnel des antipaludiques et la persistance de leur efficacité.

\section{Conclusion}

Cette enquête réalisée dans deux centres de santé de Cabinda souligne la fréquente mauvaise compréhension des examens pratiqués pour la mise en évidence de l'infection palustre et la sur-prescription de médicaments antipaludiques. Six traitements par Artemisinin Combined Therapy sur dix ont été délivrés à des enfants avec un TDR négatif. Les réponses possibles à cette situation sont avant tout du domaine de la formation des professionnels. L'éducation à la santé de la population et les incitations à une vraie mesure de réduction de la transmission du paludisme, dormir sous moustiquaire de préférence imprégnée d'insecticide, sont également nécessaires.

Liens d'intérêt : Les auteurs déclarent ne pas avoir de liens d'intérêt.

\section{Références}

1. Altaras R, Nuwa A, Agaba B, et al (2016) Why do health workers give anti-malarials to patients with negative rapid test results? A qualitative study at rural health facilities in western Uganda. Malar J 15:23 doi: 10.1186/s12936-015-1020-9

2. Beauchamp C (1988) Fièvre d'hier, paludisme d'aujourd'hui. Vie et mort d'une maladie. Annales Economies Sociétés Civilisations 43:249-25. doi: 10.3406/ahess.1988.283483

3. Direçao nacional de Saúde publica (2014) Programma nacional de controlo da malaria. Directrizes e normas de conduta para o diagnostico e tratamento da malaria. Luanda, $25 \mathrm{p}$

4. Hooft AM, Ripp K, Ndenga B, et al (2017) Principles, practices and knowledge of clinicians when assessing febrile children: a qualitative study in Kenya. Malar J 16:381. doi: 10.1186/ s12936-017-2021-7

5. Kyabayinze DJ, Asiimwe C, Nakanjako D, et al (2010) Use of RDTs to improve malaria diagnosis and fever case management at primary health care facilities in Uganda. Malar J 9:200. doi: $10.1186 / 1475-2875-9-200$ 
6. Ly AB, Tall A, Perry R, Baril L, et al (2010) Use of HRP-2-based rapid diagnostic test for Plasmodium falciparum malaria: assessing accuracy and cost-effectiveness in the villages of Dielmo and Ndiop, Senegal. Malar J 9:153. doi: 10.1186/1475-2875-9-153

7. Manguin S, Foumane V, Besnard P, et al (2017) Malaria overdiagnosis and subsequent overconsumption of antimalarial drugs in Angola: Consequences and effects on human health. Acta Trop 171:58-63. doi: 10.1016/j.actatropica.2017.03.022
8. Sayang C, Soula G, Tahar R, et al (2009) Use of a histidine-rich protein 2-based rapid diagnostic test for malaria by health personnel during routine consultation of febrile outpatients in a peripheral health facility in Yaounde, Cameroon. Am J Trop Med Hyg $81: 343-7$

9. WHO (2015) Guidelines for the treatment of malaria. Geneva, $3^{\text {rd }}$ edition, $316 \mathrm{p}$ 